\title{
Numerically Nonreflecting Boundary and Interface Conditions for Compressible Flow and Aeroacoustic Computations
}

\author{
Tim Colonius* \\ California Institute of Technology, Pasadena, California 91106
}

\begin{abstract}
Accurate nonreflecting or radiation boundary conditions are important for effective computation of aeroacoustic and compressible flow problems. The performance of such boundary conditions is often degraded upon discretization of the equations with finite difference and time marching methods. In particular, poorly resolved, spurious sawtooth waves are generated at boundaries due to the dispersive nature of the finite difference approximation. These disturbances can lead to spurious self-sustained oscillations in the flow (self-forcing), poor convergence to steady state, and long time instability of the numerics. Exact discretely nonreflecting boundary closures (boundary conditions for a downwind artificial boundary and an upwind physical boundary) are derived by considering a onedimensional hyperbolic equation discretized with finite difference schemes and Runge-Kutta time advancements. The current methodology leads to stable local finite difference-like boundary closures, which are nonreflecting to an essentially arbitrarily high order of accuracy. These conditions can also be applied at interfaces where there is a discontinuity in the wave speed (a shock) or where there is an abrupt change in the grid spacing. Compared to other boundary treatments, the present boundary and interface conditions can reduce spurious reflected energy in the computational domain by many orders of magnitude.
\end{abstract}

\section{Introduction}

$\mathbf{V}$ ARIOUS approximations have been applied to the compressible flow equations to derive artificial boundary conditions. Exact nonlocal nonreflecting boundary conditions are usually expanded to first or second order in the wave number of the coordinate parallel to the boundary to arrive at a local approximation. ${ }^{1-3}$ Expansions to higher order usually lead to unstable/ill-posed methods. Radiation boundary conditions, e.g., Ref. 4, are also usually approximate in nature, becoming exact in the limit of the domain extending to infinity. Even when an exactly nonreflecting boundary condition can be derived for the continuous partial differentialequation(PDE) (such as in applications to the Helmholtz equation), discretization of the PDE and boundary conditions leads to additional reflections, in the form of poorly resolved sawtooth waves, due to the dispersive nature of finite difference approximations. ${ }^{5,6}$ Such disturbances can lead to spurious self-sustainedoscillations in the flow (self-forcing), poor convergence to steady state, and long time instability of the numerics. For example, Tam and $\mathrm{Webb}^{7}$ recognized such a situation in applying radiation conditions to the Helmholtz equation and provided an alternative formulation of the conditions derived from the discrete equations using second-order finite differences. This method was successful at improving the nonreflectivity of the boundary even for the spurious waves, but it is not applicable to time-domain problems. Hall et al. ${ }^{8}$ derived specialized nonreflecting boundary conditions for the Euler equations discretized with a finite volume Lax-Wendroff scheme, using iterative methods to construct the discrete eigenmodes of the equations.

Here exact discretely nonreflecting boundary conditions are derived for one-dimensional linear hyperbolic equations, which are discretized with finite difference schemes. Various lowdissipation/low-dispersion finite difference schemes have been found to be very effective in aeroacoustic computations. ${ }^{9-16}$ For details the reader is referred to the recent review by Lele. ${ }^{17}$ The methodology used here is applicable to arbitrary centered finite difference schemes and time marching schemes, though we illustrate the analysis with the fourth-order compact finite difference scheme and fourth-orderRunge-Kutta time advancement.

Received Sept. 6, 1996; revision received March 14, 1997; accepted for publication March 17, 1997. Copyright 997 by Tim Colonius. Published by the American Institute of Aeronatiles and Astronautics, Inc., with permission.

*Assistant Professor of Mechanical Engineering, Division of Engineering and Applied Science. Member AIAA.
The analysis extends work on downwind boundary conditions by Vichnevetsky ${ }^{6,18}$ to compact finite difference schemes and presents new analysis for the upwind boundary conditions. In particular, the current methodology leads to stable finite difference-like boundary closure schemes, which can be derived to essentially arbitrarily high orders of accuracy. In addition, the boundary conditions naturally lead to conditions that should be imposed at the interface between flow regions where either the grid spacing is suddenly changed, or where there is a discontinuity, i.e., shock, in the wave speed. Though the boundary conditions derived here are for onedimensional equations, they can be extended to multidimensional problems; the analysis and results are involved and will be presented in future publications.

\section{Interior Differencing Scheme}

Boundary conditions are first considered for the simple first-order advection equation

$$
u_{t}+M u_{x}=0
$$

where $M>0$ is a constant. Considering the region $x \geq 0$ and $t \geq 0$, a boundary condition at $x=0$ is required:

$$
u(0, t)=f(t)
$$

An initial condition $u(x, 0)=g(x)$ with $g(0)=f(0)$ is posed at $t=0$.

Equation (1) is to be solved numerically by applying a finite difference approximation for the spatial derivative and integrating the resulting system of ordinary differential equations with an appropriate time marching scheme. The semi-infinite space $x>0$ can be made finite by imposing an artificial boundary condition at $x=L$. Let

$$
x_{j}=j h, \quad j=0,1,2, \ldots, N-1, N
$$

where $h=L / N$ is the constant grid spacing in $x$. The shorthand notation $u_{j}=u\left(x_{j}, t\right)$ is used.

Three-point central finite difference schemes are chosen for approximating $\partial u / \partial x$ :

$$
\alpha\left(\frac{\partial u}{\partial x}\right)_{j+1}+\left(\frac{\partial u}{\partial x}\right)_{j}+\alpha\left(\frac{\partial u}{\partial x}\right)_{j-1} \approx \frac{a}{h}\left(u_{j+1}-u_{j-1}\right)
$$

If $\alpha=0$ and $a=\frac{1}{2}$, Eq. (4) is the standard three-point central finite difference equation, which is second-order-accurate, i.e., the 
truncation error is $\left(h^{2}\right)$. If $\alpha=\frac{1}{4}$ and $a=\frac{3}{4}$, Eq. (4) is the Pade finite differenceapproximationand is fourth-orderaccurate. Inserting Eq. (4) into Eq. (1) gives

$$
\alpha\left(\frac{\mathrm{d} u}{\mathrm{~d} t}\right)_{j+1}+\left(\frac{\mathrm{d} u}{\mathrm{~d} t}\right)_{j}+\alpha\left(\frac{\mathrm{d} u}{\mathrm{~d} t}\right)_{j-1} \approx \frac{-M a}{h}\left(u_{j+1}-u_{j_{-1}}\right)
$$

Higher-orderaccurate and optimized ${ }^{19,20}$ schemes on larger stencils can also be analyzed in the framework subsequently presented. For brevity, only schemes on a three-point stencil are considered here.

To analyze the resolution characteristicsand boundary reflectivity of the scheme, it is useful to consider its performance in Fourier space. If $\omega$ is the real frequency and $k$ is the real wave number in $x$, then the dispersion relationship of the semidiscrete approximation [Eq. (5)] is given by

$$
\frac{\omega h}{M}=\frac{-2 a \sin (k h)}{1+2 \alpha \cos (k h)}
$$

Unlike the Fourier modes of Eq. (1), Eqs. (6) and (7) represent dispersive waves. The exact and modified dispersion relations for the semidiscrete scheme are plotted in Fig. 1a for both secondand fourth-order schemes. Note that the maximum value $k h=\pi$ corresponds to waves with only two grid points per wavelength. For dispersive systems, energy propagates at the group velocity, $V=$ - $(\partial \omega / \partial k)$. Equation (1) has a constant group velocity, $V=M$. The group velocities for Eq. (6) are plotted in Fig. 1b and show that for waves with many grid points per wavelength, i.e., small $k h$, energy travels with the (correct) group velocity of the continuous system $M$, while the poorly resolved waves (only a few points per wavelength) travel with unphysical group velocities, which become negative for the most poorly resolved waves. The waves with $V<0$ are termed spurious numerical waves after Vichnevetsky.

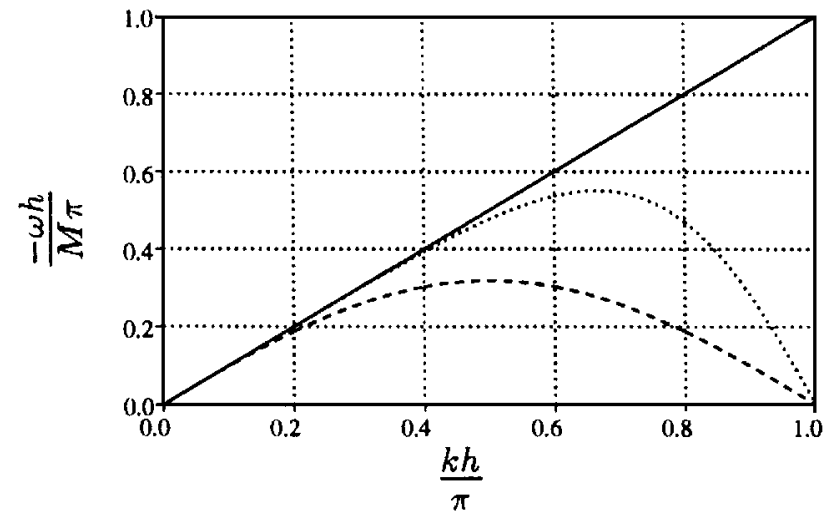

a) Modified dispersion relationship

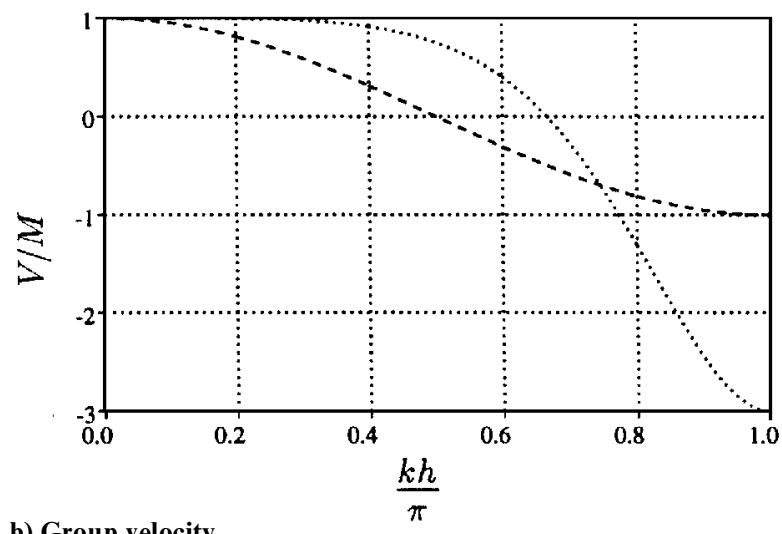

b) Group velocity

Fig. 1 Modified dispersion relationship and group velocity for solutions of Eq. (1) corresponding to - , exact solution; - - - , secondorder finite difference solution to Eq. (5); and ..., fourth-order finite difference solution to Eq. (5).
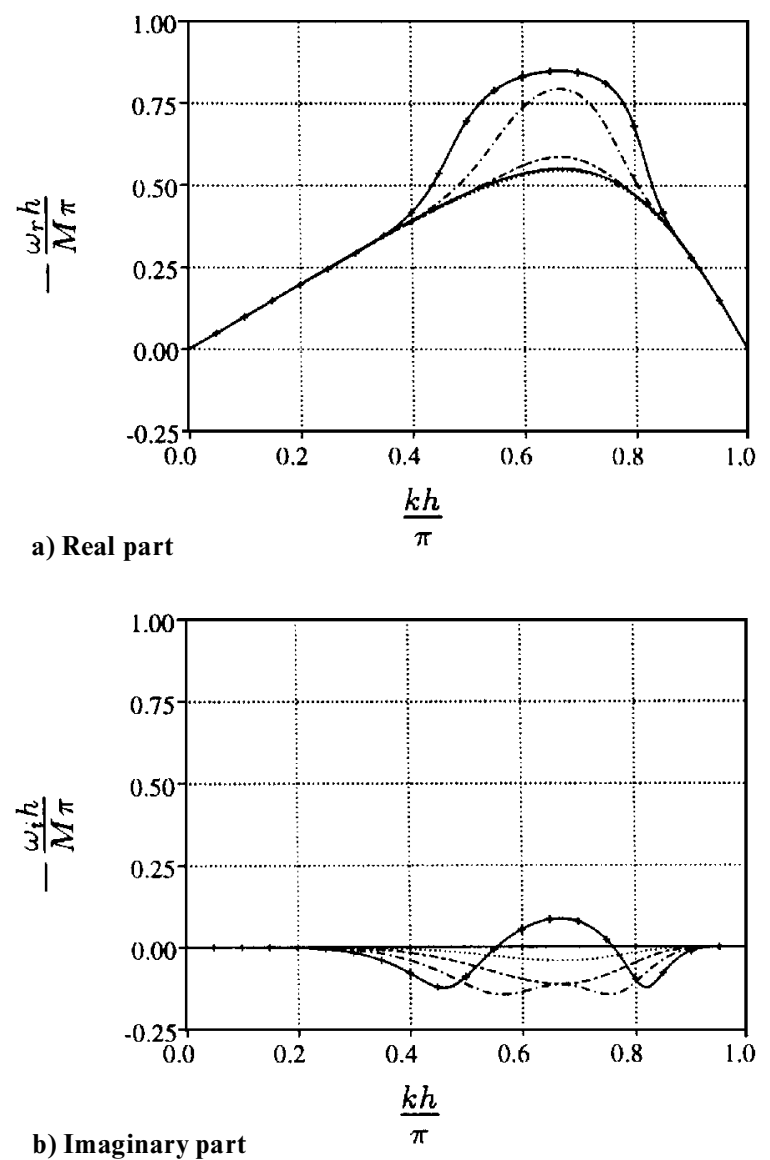

Fig. 2 Modified dispersion relationship for the fully discrete scheme at various values of $C$ : $-C=0$, semidiscrete;,$-- C=0.2 ; \ldots$, $C=1.0 ;---, C=1.25 ;-, C=1.5$; and - with,$+ C=1.75$ (note that the dashed and solid lines fall nearly on top of one another and the imaginary part for $C=0.2$ is nearly zero).

The boundary conditions developed subsequently depend explicitly on the choice of spatial differentiation scheme, i.e., the values of $\alpha$ and $a$ in Eq. (5), but can be used with any stable time advancement of Eq. (5), including optimized low dispersion/dissipation schemes. ${ }^{21}$ In subsequent examples, the fourth-order Runge-Kutta scheme is used. The fully discrete form of the dispersionrelationship for an $m$ th-order Runga-Kutta scheme is

$$
-\frac{\omega h}{M}=\frac{1}{i C} \ln \left\{\sum^{m} \frac{C^{l}}{l !}\left[\frac{2 a i \sin (k h)}{1+2 \alpha \cos (k h)}\right]^{l}\right\}
$$

where $C=M \Delta t / h$ is the Courant-Friedrichs-Lewy (CFL) number. Curves of the modified dispersion relationship for the corresponding fully discrete scheme are plotted in Fig. 2 for various values of the CFL number for the Pade scheme with $\alpha=\frac{1}{4}$. Note that the normalized frequency $\omega \Delta t$ is now complex; the real part represents the (dispersed) traveling wave components of $\tilde{u}$, whereas the imaginary part dissipates the wave. It is evident that dispersion relationship corresponding to the semidiscrete scheme [Eq. (5)] is only slightly modified by the time advancement for $C \approx 1$ and smaller. For $C>1.64$ (the Von Neumann stability limit for the third-order Runge-Kutta scheme is $C<1.0$ ), the imaginary part of the frequency becomes negative indicating instability in agreement with the Von Neumann stability analysis. ${ }^{19}$

\section{Boundary Conditions}

We now follow the method of Vichnevetsky ${ }^{6}$ for analyzing the behavior of the right-going and left-going parts of the solution near the computational boundaries at $x=0$ or 1 . Taking the Fourier transform in time of Eq. (5), we obtain

$$
b * \hat{u}_{j+1}+\hat{u}_{j}+b \hat{u}_{j-1}=0
$$




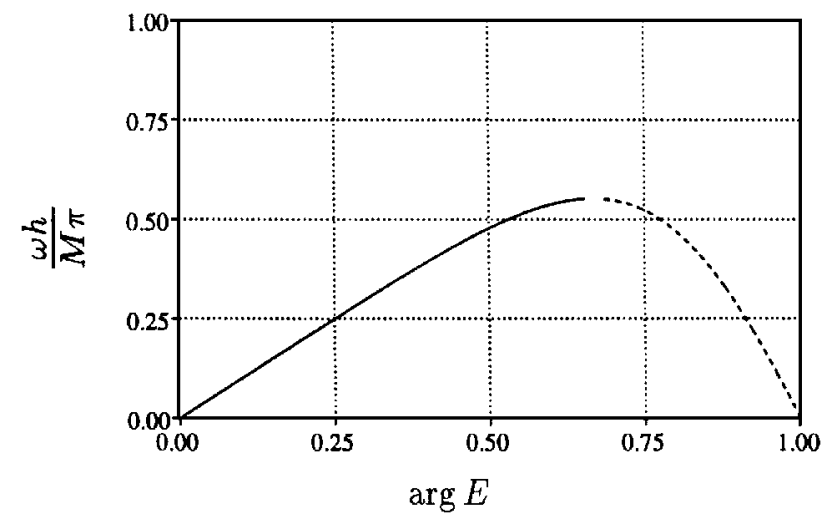

Fig. 3 Argument of the roots of Eq. (10): ..., $E^{+}$and ---, $E-$.

where $\hat{u}$ is the time Fourier transform of $u$ and where $b=\alpha+$ $(i M a / \omega h), i=1^{1}$, and $b *$ is the complex conjugate of $b$. Equation (8) can bereduced to two decoupled first-order equations by seeking solutions of the form

$$
\hat{u}_{j+1}=E(\omega) \hat{u}_{j}
$$

Substituting into Eq. (8) gives the quadratic equation

$$
b * E^{2}+E+b=0
$$

One of the roots, which we label $E^{+}$, corresponds to a consistent approximation of the wave solution to Eq. (1), whereas the other root, $E-$, is entirely spurious. ${ }^{6}$ It can be shown for frequencies less than some cutoff $\omega_{c}$ that both roots have magnitude 1 , which means that each wave propagates in $x$ without dissipation. This property holds for any central difference scheme, but different schemes have different cutoff frequencies. It can also be shown that if the boundary condition $f(t)$ does not contain energy at frequencies higher than $\omega_{c}$, then waves with frequency higher than $\omega_{c}$ cannot exist in the numerical solution. ${ }^{6}$

For $\alpha=\frac{1}{4}$, the roots are given by

$$
E \pm=\frac{-2 i z+\overline{9} /-3 z^{2}}{i z+\sqrt{3}}
$$

where $z=\omega h / M$ and the cutoff frequency is given by $z_{c}=$ $\omega_{c} h / M=3$

The phase speed of the wave corresponding to each root of Eq. (10) is plotted in Fig. 3 for the fourth-orderscheme with $\alpha=\frac{1}{4}$. The phase speed is given by the argument of $E$. For $\omega<\omega_{c}$, the two curves make up the modified semidiscretedispersion relation shown in Fig. 1a, separated into solutions that have positive group velocity $E^{+}$and those with negative group velocity $E^{-}$. For $\omega>\omega_{c}$, the roots do not correspond to traveling waves, and their group velocity is not plotted.

Following Vichnevetsky, ${ }^{6}$ we can separate our solution, $\hat{u}$, at any point in the interior into its right-going and left-going parts by writing

where

$$
\hat{u}_{j}=\hat{u}_{j}^{+}+\hat{u}_{j}^{-}
$$

$$
\begin{aligned}
& \hat{u}_{j+1}^{+}=E^{+} \hat{u}_{j}^{+} \\
& \hat{u}_{j+1}^{-}=E^{-} \hat{u}_{j}^{-}
\end{aligned}
$$

\section{A. Downwind Boundary (Artificial Boundary)}

Consider the boundary at $x=1$ where Eq. (5) cannot be used. Although there is no physical boundary condition at $x=1$, a numerical boundary condition must be specified. Because the modes in the interior are decoupled, we can write a perfectly numerically nonreflecting boundary condition

$$
\hat{u}_{N}=0
$$

(also given as a "numerical Sommerfeld boundary condition" in Ref. 18), which is equivalent to

$$
\hat{u}_{N}=E^{+} \hat{u}_{N-1}
$$

because $1 \_\left(E^{+} / E^{-}\right) \neq 0$.
To apply Eq. (16) in a computation, we must first take its inverse Fourier transform in time. The mode $E^{+}$does not depend linearly on $\omega$, because it contains the square root, the resulting inverse transform of Eq. (16) is nonlocal in time. This situation is similar to that which arises in developing nonreflecting boundary conditions for multidimensional equations as first noted by Enquist and Majda. ${ }^{1}$ To arrive at a boundary condition that is local in time, $E^{+}$can be expanded in a Taylor's series (other expansions are possible) about $\omega=0$ (equivalently $z=0$ ). The series is truncating at a particular order. Then the inverse Fourier transform gives a local boundary condition in the form of an equation for the evolution of $u_{N}$ in terms of $u_{N_{-} 1}$ and their time derivatives.

Alternatively, a more general local boundary condition for the point $u_{N}$ of the form

$$
\sum_{d}^{N_{a}} a_{k}\left(\frac{h}{M}\right)^{k} \frac{\mathrm{d}^{k} u_{N}}{\mathrm{~d} t^{k}}+\beta \frac{h}{M} \frac{\mathrm{d} u_{N_{-}}}{\mathrm{d} t}=\sum_{d}^{N_{b}} b_{k} u_{N_{-} k}
$$

where $N_{a}$ and $N_{b}$ represent the highest time derivative of the boundary scheme and the maximal stencil width at the boundary, respectively. The constants $a_{k}, b_{k}$, and $\beta$ are chosen to make the boundary condition nonreflecting, as discussed subsequently. The rationale for the form of Eq. (17) will be discussed shortly. First, we take the time Fourier transform of Eq. (17) to obtain

$$
\hat{u}_{N}\left[\sum_{d}^{N_{a}} a_{k}(i z)^{k}\right]+\beta i z \hat{u}_{N_{-} 1}=\sum_{d}^{N_{b}} b_{k} \hat{u}_{N_{-} k}
$$

Now, we let $\hat{u}=\hat{u}^{+}+\hat{u}^{-}$in Eq. (18) to obtain

$$
\hat{u}_{N}^{+} A(z)=\hat{u}_{N} B(z)
$$

where

$$
\begin{gathered}
A(z)=\frac{\beta i z}{E^{+}}+\sum_{d}^{N_{a}} a_{k}(i z)^{k}-\sum_{d}^{N_{b}} \frac{b_{k}}{E^{+k}} \\
B(z)=\frac{-\beta i z}{E-}-\sum_{a}^{N_{a}} a_{k}(i z)^{k}+\sum_{d}^{N_{b}} \frac{b_{k}}{E-^{k}}
\end{gathered}
$$

which gives the desired relation for the coupling between the leftgoing and right-going modes at the boundary.

Again, the exact boundary condition is that $\hat{u}_{N}=0$. To obtain an appropriate boundary condition, therefore, we choose the coefficients $a_{k}$ and $b_{k}$ and $\beta$ to minimize $A(z)$ subject to producing a stable boundary condition (stability is discussed in Sec. III.C).

The form of Eq. (17) is expanded from the one considered in Ref. 18 to include the possibility of higher derivatives (with respect to time) on the left-hand side and the inclusion of $\mathrm{d} u / \mathrm{d} t$ at the point $N \_1$ adjacent to the boundary. The terms included in Eq. (17) are the most general ones that can be used without destroying the tridiagonal structure of the Pade differencing scheme, and Eq. (17) can be easily implemented into the numerical scheme by rewriting it as a system of first-order equations.

There are several possible strategies for choosing $a_{k}$ and $b_{k}$ to minimize $A(z)$. For example, one can, as we do here, expand $A(z)$ in a Taylor's series about $z=0$ and set the coefficients $a_{k}$ and $b_{k}$ to drive each term in the expansion to zero. It would also be possible to minimize a global measure of $A(z)$, such as its $L 2$ norm over the pertinent range of frequencies, $0<z<z_{c}$. Moreover, the coefficients could be chosen to minimize the reflections at a particularvalue of $z$. Finally, a combination of these strategies could be used.

For the fourth-orderscheme, $\alpha=\frac{1}{4}$, a number of possibleschemes embodied by Eq. (17) are tabulated in Table 1. As discussed in Sec. III.C, all schemes are derived for the fourth-order Pade-scheme $\left(\alpha=\frac{1}{4}\right)$. Unless otherwise noted, any combination of the boundary conditions are stable for the fourth-order Runge-Kutta time advancement with maximum CFL determined by the interior scheme alone. In each case, the coefficients are chosen by forcing terms in the Taylor's series of $A(z)$ to zero up to a particular order of accuracy, $\left(\mathcal{O}^{n}\right)$. This order is indicated in Table 1 . Note that for the 
Table 1 Coefficients for downwind boundary conditions for the point $u_{N}$ (see Sec. III.A)

\begin{tabular}{|c|c|c|c|c|c|c|c|c|c|c|}
\hline $\begin{array}{l}\text { Scheme: } \\
\text { Order: }\end{array}$ & $\begin{array}{c}1.1 \\
2\end{array}$ & $\begin{array}{c}1.2 \\
3\end{array}$ & $\begin{array}{c}2.1 \\
3\end{array}$ & $\begin{array}{c}2.1 \beta \\
4\end{array}$ & $\begin{array}{c}3.1 \\
4\end{array}$ & $\begin{array}{c}4.1 \\
5\end{array}$ & $\begin{array}{c}3.2 \beta \\
6\end{array}$ & $\begin{array}{c}6.1 \\
7\end{array}$ & $\begin{array}{c}6.3 \\
9\end{array}$ & $\begin{array}{c}6.4 \beta \\
11\end{array}$ \\
\hline$\beta$ & 0 & 0 & 0 & 4 & 0 & 0 & 26 & 0 & 0 & $4,088,016$ \\
\hline$a_{1}$ & 1 & 2 & 2 & 2 & 6 & 12 & 16 & 72 & 26,388 & $1,725,840$ \\
\hline$a_{2}$ & 0 & 1 & 0 & 0 & 0 & 0 & 2 & 0 & 8,640 & 259,704 \\
\hline$a_{3}$ & 0 & 0 & 0 & 0 & 0 & 0 & 0 & 0 & 1,348 & 65,880 \\
\hline$a_{4}$ & 0 & 0 & 0 & 0 & 0 & 0 & 0 & 0 & 0 & 8,112 \\
\hline$b_{0}$ & -1 & 2 & -3 & -5 & -11 & 25 & -34 & -175 & $-33,514$ & $-4,006,835$ \\
\hline$b_{1}$ & 1 & 2 & 4 & 4 & 18 & 48 & 25 & 424 & 44,728 & $1,756,548$ \\
\hline$b_{2}$ & 0 & 0 & -1 & 1 & -9 & -36 & 10 & -521 & $-17,345$ & $2,782,179$ \\
\hline$b_{3}$ & 0 & 0 & 0 & 0 & 2 & 16 & -1 & 456 & 8,780 & $-633,008$ \\
\hline$b_{4}$ & 0 & 0 & 0 & 0 & 0 & -3 & 0 & -253 & $-3,334$ & 114,507 \\
\hline$b_{5}$ & 0 & 0 & 0 & 0 & 0 & 0 & 0 & 80 & 764 & $-14,292$ \\
\hline$b_{6}$ & 0 & 0 & 0 & 0 & 0 & 0 & 0 & -11 & -79 & 901 \\
\hline
\end{tabular}

fourth-order interior scheme $\left(\alpha=\frac{1}{4}\right)$, the first three terms in the series for $A(z)$ vanish:

$$
A(0)=0,\left.\quad \frac{\mathrm{d} A}{\mathrm{~d} z}\right|_{z=0}=0, \quad \text { and }\left.\quad \frac{\mathrm{d}^{2} A}{\mathrm{~d} z^{2}}\right|_{z=0}=0
$$

independently of $\alpha$. For these cases, the schemes are equivalent to a discrete approximation to an upwind approximation to $\partial u_{N} / \partial x$ (examples are schemes 1.1, 1.2, 2.1, 2.1 $\beta$, and 3.1 in Table 1).

Scheme 6.1 is similar to the numerical Sommerfeld boundaryconditions of Vichnevetsky, ${ }^{18}$ except that it applies to the Pade interior scheme with $\alpha=\frac{1}{4}$ rather than the explicit second-order $(\alpha=0)$ interior scheme. When $N_{a}=0$ in Eq. (17), i.e., there are no time derivatives used in the boundary conditions, the present boundary conditions are similar to extrapolation boundary conditions sometimes used to treat point $u_{N}$. For example, when $N_{b}=1$, Eq. (17) gives $u_{N}=u_{N_{-} 1}$ to $\left(b^{z}\right)$, which is equivalent to a zeroth-order extrapolation. When $N_{b}=2$, Eq. (17) gives the standard first-order extrapolation, $u_{N}=2 u_{N_{-} 1}+u_{N_{-2}}$, which is accurate to $O\left(z^{2}\right)$.

The boundary schemes given in Table 1 are all stable when applied to the fourth-order interior $\left(\alpha=\frac{1}{4}\right)$ scheme with a maximum allowable CFL number equal to the maximum CFL number from the Von Neumann stability analysis of the interior finite difference scheme. The stability analysis is given in Sec. III.C. There is no apparent limit to the order of accuracy that can be obtained with this approach. This is in contrast to boundary closures found by approximating Eq. (1) with upwind finite difference schemes, where it is very difficult to find schemes that are stable and for which the order of accuracy is not smaller than that of the interior scheme (see, for example, Ref. 22). Thus, the order of the nonreflectivity of such upwind schemes is limited to the order of accuracy of the interior scheme.

Numerical tests of several of these boundary conditions are given in Sec. III.D and show that the long time behavior of the solution is greatly improved by using the highest-order boundary closure possible.

\section{B. Upwind Boundary (Physical Boundary)}

At $x=0$, the physical boundary condition $u(0, t)=f(t)$ is given. The most obvious way to implement this boundary condition is to use $f(t)$ and $(\partial f / \partial x)(t)$ to close the Pade derivative scheme at $x=0$. This direct implementation is actually highly reflective for the poorly resolved waves that propagate upstream and strike the upwind boundary. ${ }^{23}$ This follows by considering the decomposition of the solution (Fourier transformed in time) into its left- and rightgoing modes at the point $u_{0}$,

$$
\hat{u}_{0}=\hat{u}_{0}^{+}+\hat{u}_{0}^{-}=\hat{f}
$$

For example, if $\hat{f}=0$, then any left-going portion of the solution $\hat{u}^{-}$, is perfectly reflected into a right-going solution $\hat{u}^{+}$. Instead, Vichnevetsky ${ }^{6}$ proposed

$$
u_{0}=2 f(t)-u_{1}+(6 z)
$$

and

$$
\left.u_{0}=4 f(t)-2 u_{1}-u_{2}+\mathcal{O}^{z^{2}}\right)
$$

but he gave no general method to construct higher-order approximations. As we will show, Eq. (24) is actually only correct to $(6 z)$.

Consider the following boundary condition written in Fourier space:

$$
\hat{u}_{0}=\hat{f}\left[1-\left(E^{+} / E^{-}\right)\right]+\left(\hat{u}_{1} / E^{-}\right)
$$

Then decomposing into right- and left-going modes, $\hat{u}^{+}$and $\hat{u}^{-}$ gives (because $\left.\left[1-\left(E^{+} / E^{-}\right)\right] \neq 0\right)$

$$
\hat{u}_{+}=\hat{f}
$$

which is the upwind exactly numerically nonreflecting counterpart of Eq. (15) from the last section. Motivated by the form of the downwind boundary condition [Eq. (17)], a general boundary condition for $u_{0}$ is written

$$
\sum_{c}^{N_{c}} c_{k}\left(\frac{h}{M}\right)^{k} \frac{\mathrm{d}^{k} u_{0}}{\mathrm{~d} t^{k}}+\beta \frac{h}{M} \frac{\mathrm{d} u_{1}}{\mathrm{~d} t}=\phi(t)+\sum_{d}^{N_{d}} d_{k} u_{k}
$$

where the function $\phi(t)$ is subsequently defined in Eq. (31). Taking the Fourier transform of Eq. (27) and using $\hat{u}=\hat{u}^{+}+\hat{u}^{-}$gives

$$
\hat{u}_{0}^{+} C(z)=\hat{u}_{0}^{-} D(z)+\hat{\phi}
$$

where

$$
\begin{gathered}
C(z)=\beta i z E^{+}+\sum_{d}^{N_{c}} c_{k}(i z)^{k}-\sum_{d}^{N_{d}} d_{k} E^{+k} \\
D(z)=-\beta i z E^{-}-\sum_{d}^{N_{c}} c_{k}(i z)^{k}+\sum_{d}^{N_{d}} d_{k} E^{-k}
\end{gathered}
$$

To obtain an approximate boundary condition, therefore, the coefficients $c_{k}$ and $d_{k}$ should be chosen to zero successive terms in the Taylor's series of $D(z)$ about $z=0$. Then if we define

$$
\hat{\phi}=C(z) \hat{f}
$$

we recover $\hat{u}_{+}=\hat{f}$ to an arbitrary order of accuracy. Note that Eq. (31) is nonlocalbecause $C(z)$ is not a polynomialin $z$. Therefore, $C(z)$ should also be expanded in a Taylor's series to the same order as the expansion of $D(z)$. Interestingly,this indicates the high-order accurate boundary conditions for the point $u_{0}$ require information about high time derivatives of the forcing function $f(t)$. This may be cumbersome in practice. However, two common situations, when $f(t)=0$ and when $f(t)$ is composed of harmonics, present no difficulties.

Expanding Eq. (31) in a Taylor's series gives the local approximation for $\phi(t)$,

$$
\phi(t)=\sum_{d}^{N_{0}} f_{k}\left(\frac{h}{M}\right)^{k} \frac{\mathrm{d}^{k} f(t)}{\mathrm{d} t^{k}}
$$

where $N_{0}$ is the order of the last term in the Taylor's series of $D(z)$, which is forced to zero by choosing the coefficients $c_{k}$ and $d_{k}$, i.e., the order of the boundary condition minus one. 
Table 2 Coefficients for upwind boundary conditions for the point $u_{0}$ (see Sec. III.B)

\begin{tabular}{|c|c|c|c|c|c|c|c|c|c|c|}
\hline $\begin{array}{l}\text { Scheme: } \\
\text { Order: }\end{array}$ & $\begin{array}{c}1.1 \\
2\end{array}$ & $\begin{array}{c}1.2^{\mathrm{a}} \\
3\end{array}$ & $\begin{array}{c}2.1 \\
3\end{array}$ & $\begin{array}{c}2.1 \beta \\
4\end{array}$ & $\begin{array}{c}3.1 \\
4\end{array}$ & $\begin{array}{c}4.1 \\
5\end{array}$ & $\begin{array}{c}3.2 \beta \\
6\end{array}$ & $\begin{array}{c}6.1 \\
7\end{array}$ & $\begin{array}{c}6.3 \\
9\end{array}$ & $\begin{array}{c}6.4 \beta \\
11\end{array}$ \\
\hline$\beta$ & 0 & 0 & 0 & -4 & 0 & 0 & 14 & 0 & 0 & 105,072 \\
\hline$c_{1}$ & -1 & $\_^{6}$ & $ـ^{2}$ & $ـ^{2}$ & $\_^{2}$ & $ـ_{4}^{4}$ & $\_8$ & $\_8$ & _53,244 & 35,184 \\
\hline$c_{2}$ & 0 & $-^{1}$ & 0 & 0 & 0 & 0 & -2 & 0 & $-12,240$ & 1,992 \\
\hline$c_{3}$ & 0 & 0 & 0 & 0 & 0 & 0 & 0 & 0 & _1,372 & 360 \\
\hline$c_{4}$ & 0 & 0 & 0 & 0 & 0 & 0 & 0 & 0 & 0 & 32 \\
\hline$d_{0}$ & 3 & 18 & 9 & 3 & 15 & 45 & 66 & 189 & 256,932 & 53,901 \\
\hline$d_{1}$ & 3 & 18 & 12 & 12 & 30 & 120 & 93 & 792 & 556,632 & _90,684 \\
\hline$d_{2}$ & 0 & 0 & 3 & 9 & 21 & 132 & 54 & 1,539 & 611,955 & $-164,025$ \\
\hline$d_{3}$ & 0 & 0 & 0 & 0 & 6 & 72 & 27 & 1,704 & 457,380 & _ 58,320 \\
\hline$d_{4}$ & 0 & 0 & 0 & 0 & 0 & 15 & 0 & 1,095 & 181,116 & _ 50,301 \\
\hline$d_{5}$ & 0 & 0 & 0 & 0 & 0 & 0 & 0 & 384 & 36,612 & _ 14,580 \\
\hline$d_{6}$ & 0 & 0 & 0 & 0 & 0 & 0 & 0 & 57 & 621 & $-3,159$ \\
\hline$f_{0}$ & ${ }^{6}$ & _36 & 24 & _24 & ${ }_{-72}$ & $\_384$ & 240 & _5,760 & $2,101,248$ & 327,168 \\
\hline$f_{1}$ & 2 & 12 & 16 & 24 & 88 & 656 & 288 & 15,616 & $4,010,688$ & _ 746,496 \\
\hline$f_{2}$ & 0 & -10 & $-^{12}$ & -20 & $\_84$ & -768 & 292 & 25,728 & $\__{5,490,432}$ & $1,174,272$ \\
\hline$f_{3}$ & 0 & 0 & 0 & 12 & 60 & 680 & 216 & 31,584 & $5,682,560$ & $1,397,376$ \\
\hline$f_{4}$ & 0 & 0 & 0 & 0 & 0 & _496 & $-400 / 3$ & -31,568 & _4,893,696 & $1,379,296$ \\
\hline$f_{5}$ & 0 & 0 & 0 & 0 & 0 & 0 & 72 & 26,992 & $3,655,800$ & $-1,176,768$ \\
\hline$f_{6}$ & 0 & 0 & 0 & 0 & 0 & 0 & 0 & _61,216/3 & $2,444,256$ & $2,681,264 / 3$ \\
\hline$f_{7}$ & 0 & 0 & 0 & 0 & 0 & 0 & 0 & 0 & $1,496,952$ & _617,328 \\
\hline$f_{8}$ & 0 & 0 & 0 & 0 & 0 & 0 & 0 & 0 & $\_854,748$ & $3,548,224 / 9$ \\
\hline$f_{9}$ & 0 & 0 & 0 & 0 & 0 & 0 & 0 & 0 & 0 & $\_235,872$ \\
\hline$f_{10}$ & 0 & 0 & 0 & 0 & 0 & 0 & 0 & 0 & 0 & $3,607,184 / 27$ \\
\hline
\end{tabular}

${ }^{\mathrm{a}}$ Upwind scheme 1.2 requires $C<0.9$.

In Table 2, the coefficients $c_{k}, d_{k}$, and $f_{k}$ are given for upwind boundary schemes similar to those presented in Table 1 for the downwind boundary. Note that if $N_{c}=0$ and $N_{d}=1$ in Eq. (27), the first-order accurate boundary condition of Vichnevetsky [Eq. (23)] is recovered. However, for $N_{c}=0$ and $N_{d}=2$, the correct version of Eq. (24) is

$$
\left.u_{0}=4 f(t)-4 \frac{h}{M} \frac{\mathrm{d} f(t)}{\mathrm{d} t}-2 u_{1}-u_{2}+\mathcal{C O}^{z^{2}}\right)
$$

\section{Stability}

The stability of the interior scheme, with periodic boundary conditions, can be analyzed using a Von Neumann stability analysis. However, the imposition of upwind and downwind boundary conditions can alter the stability characteristics of the scheme. Under the group velocity interpretation of Trefethen, ${ }^{24}$ the interior scheme plus boundary conditions will be stable unless there is spontaneous radiation of energy from the boundary into the interior. This interpretation is consistent with the Gustafsson-Kreiss-Sundström (GKS) stability criteria. ${ }^{25}$ GKS stability refers to whether or not there are eigenvalues that correspond to solutions that grow without bound (in a suitably chosen norm) in time. For a particular semidiscrete scheme found to be GKS stable, it is further required that none of the eigenvalues fall outside the stability region (in the complex $\omega$ plane) for the particular explicit time marching method employed.

Let

$$
\begin{aligned}
& \rho_{d}=|A(z) / B(z)| \\
& \rho_{u}=|D(z) / C(z)|
\end{aligned}
$$

where the subscripts $d$ and $u$ refer to the downwind and upwind boundaries, respectively. In terms of the reflection coefficients $\rho$, stability requires that there exist no values of $\omega$ in the lower-half of the complex plane (including the real axis) for which both $\rho$ is infinite and for which $E^{+} \neq 0$ at a downwind boundary or for which $E-\not 0$ at an upwind boundary. Note that this stability criteria is slightly different than that employed by Vichnevetsky. ${ }^{6}$

This criterion has been applied to each of the boundary conditions discussed in the last two sections coupled to the interior scheme with $\alpha=\frac{1}{4}$. To search for values of $\omega$ for which $\rho$ becomes infinite, the function $1 / \rho(z)$ was constructed for each scheme in Table 2. For the low-order schemes, the zeros of $1 / \rho$ can be determined analytically using Mathematica, ${ }^{26}$ whereas for the high-order schemes the zeros where found graphically by plotting $\rho$. In all cases the schemes given in Tables 1 and 2 were found to be GKS stable. As a double check of the root finding, the stability of the scheme was also

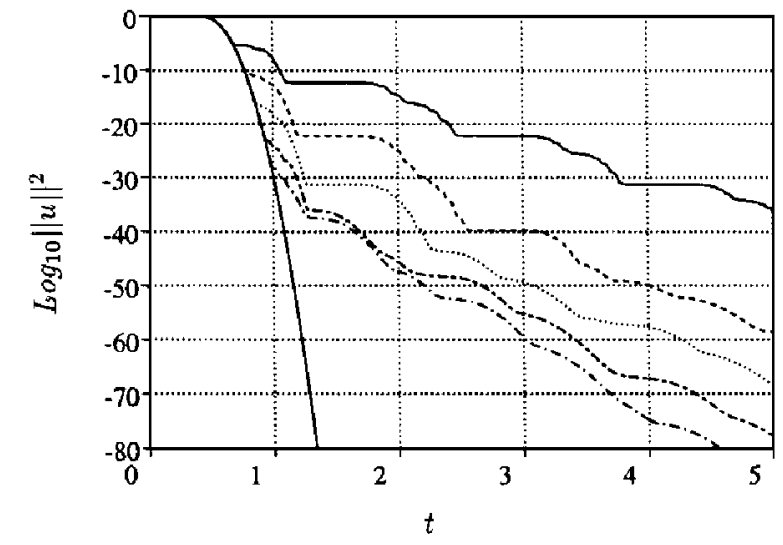

Fig. 4 Energy left in the computational domain as a function of time for various boundary conditions of Tables 1 and $2, N=200$ and $C=1$ : $\ldots$, scheme $1.1 ;---$, scheme $3.1 ; \ldots$, scheme $3.2 \beta ;---$, scheme 6.3 ; - .-, scheme $6.4 \beta$; and - , exact solution.

checked by numerically analyzing the eigenvalues of the complete (interior plus boundary conditions) semidiscrete numerical scheme with varying total numbers of node points. In that case, if any eigenvalue has a positive imaginary part, then the scheme is unstable. ${ }^{27}$ This numerical procedure was also used to find the stability limits when fourth-orderRunge-Kutta (RK4) time advancement is applied to the semidiscrete equations. For all but upwind scheme 2.1, the maximum CFL is given by the Von Neumann stability analysis of the corresponding periodic interior scheme, e.g., $C<1.64$ for RK4 time advancement and $\alpha=\frac{1}{4}$.

\section{Numerical Tests of the Boundary Conditions}

Equation (1) is now solved numerically with the fourth-order Pade scheme $\left(\alpha=\frac{1}{4}\right)$ and RK4 time marching using the boundary conditions derived in the preceding sections. The test problem is the propagation of a Gaussian pulse

$$
u(x, 0)=\exp \left[-128\left(x-\frac{1}{2}\right)^{2}\right]
$$

initially centered on a domain with $L=1$ and $N=200$. This extremely fine resolution ( 50 points across the pulse) is chosen so that errors associated with the boundary conditions can be isolated from truncation errors for the interior points. Figure 4 shows the 


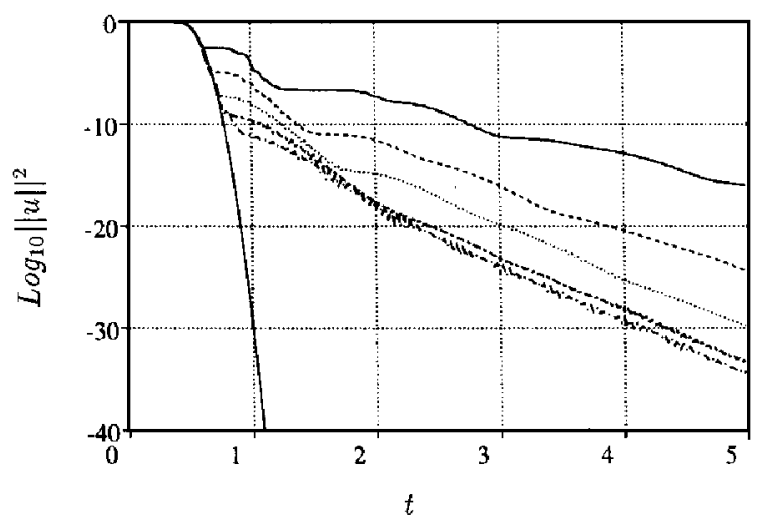

Fig. 5 Same as Fig. 4, but with $N=40$.

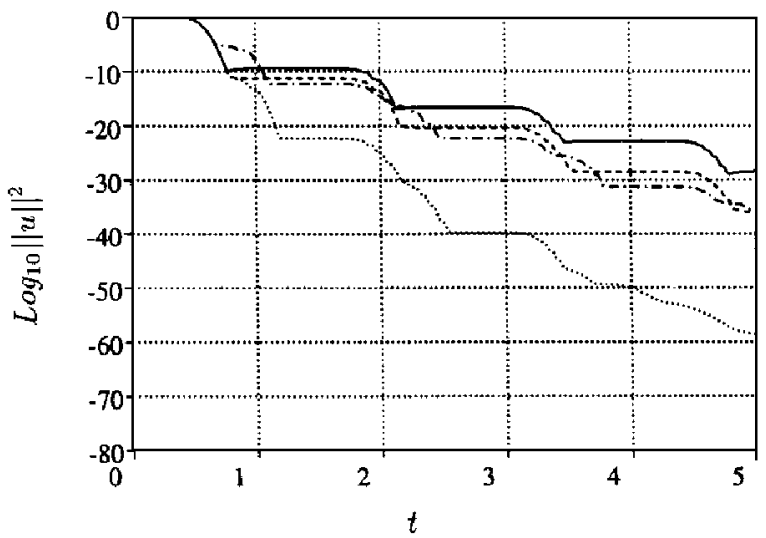

Fig. 6 Comparison of standard and present schemes: - , standard method $A ;---$, standard method $B$; --- , present scheme 1.1 ; and .... present scheme $2.1 \beta$.

results for several different boundary schemes of Tables 1 and 2 with $C=1$. Plotted against time is the $L 2$ norm of the velocity,

$$
\|\mu\|^{2}=\frac{1}{N} \sum_{j}^{N} u_{j}^{2}
$$

normalized by its initial $(t=0)$ value. Note the log scale in the plot. The pulse starts in the center of the computational domain, and the incoming wave at $x=0$ is set to zero. The staircase pattern of the plots is related to the repeated reflections of the pulse at the downwind and upwind boundary. The exact solution is also plotted for comparison. The plot shows the efficacy of the new boundary conditions at reducing reflections of the initial pulse at $x=1$ and subsequentreflections at the upwind and downwind boundaries. The 11 th-order scheme $6.4 \beta$ reduces the energy reflected by the initial pulse by many orders of magnitude from the first-order scheme.

Because the boundary conditions work by minimizing the reflection coefficients in Taylor series about $z=0$, energy at the highest frequencies has the highest reflection coefficient and, therefore, remains the longest in the computational domain. At low CFL number, this energy remains in the computational domain, unable to escape and only very slowly dissipated because for low CFL number the scheme is nearly nondissipative. By contrast, at CFL numbers near 1 and larger, the energy at the highest frequencies left by the boundary conditions is dissipated, because the Runge-Kutta time advancement has a small dissipation associated with wavelengths that correspond to the highest frequencies (see Fig. 2b). Thus, a certain amount of dissipation, at the highest frequencies, is actually beneficial in the computation.

Note that the fourth-order Pade scheme can be accurately used with much coarser grid spacing than was used in the earlier example. In Fig. 5 results are shown for the test case of Fig. 4 but with $N=$ 40 (about 10 points across the Gaussian). The conclusions about the efficacy of the boundary conditions are the same as with $N=$ 200 , though the total energy removed by any boundary condition is smaller for this case because a larger fraction of the energy is contained in highest frequencies.
In Fig. 6, the present schemes 1.1 and $2.1 \beta$ are compared to two standard ways of handling boundary conditions for the Pade finite difference scheme. The curve labeled as method A results when the physical boundary condition at $x=0$ is implemented by requiring $u_{0}=0$ and $\partial u / \partial x_{0}=0$. Method $\mathrm{B}$ results when thirdorderaccurate Pade downwind differences ${ }^{19}$ are used to approximate the derivative at $x=0$ in computing $\partial u / \partial x$ and subsequently $u_{0}$ is set to zero in each sweep of the Runge-Kutta time advancement. At the downwind boundary, methods A and B both use upwind Pade finite differences to evaluate $\partial u / \partial x_{N}$. Note that these downwind treatments are equivalentto the present downwind boundaryscheme $2.1 \beta$. Clearly, the new schemes are much more effective at removing spurious energy from the computational domain.

\section{Interface Conditions}

The boundary conditions derived in the last section are also useful at an interface between regions with different grid spacings and/or different advection velocities (wave speeds). The latter condition is a simple model for the propagation of linear disturbances through a shock wave. The advantages of allowing for dissimilar grid spacing in two separate regions of the computation are obvious. Changes in grid spacing are usually accomplished by either mapping a physical domain with smoothly varying grid spacing to a computational grid with uniform spacing or by deriving finite difference formulas (Pade or otherwise) that allow for variable grid spacing. In either case, when well-resolved waves propagate through the variable grid spacing poorly resolved sawtooth waves are reflected. ${ }^{3,28}$ More harmfully, when the sawtooth waves propagate through the variable grid spacing, they are reflected as smooth waves indistinguishable from the underlying smooth solution to the original PDE. Cain and $\mathrm{Bush}^{28}$ have studied the effects of variable grid spacing on the stability and accuracy of finite difference approximations and proposed differencing schemes that are superior in these regards. Here we consider a different approach.

Consider a one-dimensional domain with $N_{1}+1$ grid points in a region $0<x<L_{1}$ to the left of the interface and $N_{2}+1$ grid points in a region $x=L_{1}$ to $x=L_{2}$ to the right of the interface. The grid spacings are $h_{1}=L_{1} / N_{1}$ and $h_{2}=\left(L_{2}-L_{1}\right) / N_{2}$ on the left- and right-hand sides, respectively. The model equation is the same as Eq. (1) but with $M$ given by

$$
M= \begin{cases}M_{1} & \text { for } 0 \leq x<L_{1} \\ M_{2} & \text { for } L_{1} \leq^{x} \leq L_{2}\end{cases}
$$

Let $u_{j}=u\left(j h_{1}, t\right)$ for $j=0,1, \ldots, N_{1}$ on the left-hand side and $v_{j}=u\left(L_{1}+j h_{2}, t\right)$ for $j=0,1, \ldots, N_{2}$ on the right-hand side. The fourth-order Pade scheme is used on both sides of the interface. Any of the upwind boundary conditions of Table 2 can be used to determine $u_{0}$ and any of the downwind conditions of Table 1 for the point $v_{N_{2}}$. For the point $u_{N_{1}}$, the smooth solutions $\hat{u}_{N_{\Lambda}}^{+}$should pass through the interface, whereas the spurious solutions $\hat{u}_{N}$ should be zero. Thus, at point $u_{N_{1}}$, any of the downwind conditions for Table 1 can be used.

The exact interface condition for the point $v_{0}$ is, in Fourier space, that $\hat{v}_{0}^{+}=\hat{u}_{N}^{+}$and that $\hat{v}_{0}^{-}=0$. That is, the smooth waves on the right should be forced by the smooth solutions from the left, while at the same time upstream propagating spurious solutions on the left should not be reflected. By analogy with Eq. (27), a general interface condition for $v_{0}$ can be written

$$
\sum_{\mathrm{N}}^{N_{c}} c_{k}\left(\frac{h_{2}}{M_{2}}\right)^{k} \frac{\mathrm{d}^{k} v_{0}}{\mathrm{~d} t^{k}}+\beta \frac{h_{2}}{M_{2}} \frac{\mathrm{d} v_{1}}{\mathrm{~d} t}=\psi(t)+\sum_{d}^{N_{d}} d_{k} v_{k}
$$

where the forcing $\psi(t)$ is given by

$$
\psi(t)=\sum^{N g} g_{k}\left(\frac{h_{1}}{M_{1}}\right)^{k} \frac{\mathrm{d}^{k} u_{N_{1}}}{\mathrm{~d} t^{k}}+\sum_{d}^{N_{h}} h_{k} u_{N_{1} \_k}
$$

Decomposing $v_{j}$ and $u_{j}$ into left- and right-going components, the Fourier transform (in time) of Eq. 38 is

$$
C\left(z_{2}\right) \hat{v}_{0}^{+}=D\left(z_{2}\right) \hat{v}_{0}^{-}+\left[G\left(z_{1}\right)+\frac{A\left(z_{1}\right) H\left(z_{1}\right)}{B\left(z_{1}\right)}\right] \hat{u}_{N_{1}}^{+}
$$


Table 3 Coefficients for the interface condition for $v_{0}$ (see Sec. IV)

\begin{tabular}{ll}
\hline \hline Scheme 1.1 & $g_{1}=2 r$ \\
& $h_{0}=-6$ \\
Scheme 4.1 & $g_{1}=656 r+2,816 r^{2}+4,080 r^{3}+1,984 r^{4}$ \\
& $h_{0}=-384+10,880 r^{2} / 3+6,800 r^{3}+10,912 r^{4} / 3$ \\
& $h_{1}=-4,608 r^{2}-10,200 r^{3}-5,952 r^{4}$ \\
& $h_{2}=1,152 r^{2}+4,080 r^{3}+2,976 r^{4}$ \\
& $h_{3}=-512 r^{2} / 3-680 r^{3}-1,984 r^{4} / 3$ \\
Scheme 6.1 & $g_{1}=\left(171,776 r+1,389,312 r^{2}+4,263,840 r^{3}+6,439,872 r^{4}+4,858,560 r^{5}+1,469,184 r^{6}\right) / 11$ \\
& $h_{0}=\left(-190,080+6,309,792 r^{2}+24,706,584 r^{3}+40,880,560 r^{4}+32,309,424 r^{5}+10,039,424 r^{6}\right) / 33$ \\
& $h_{1}=\left(-98,280,906 r^{2}-45,101,952 r^{3}-81,477,008 r^{4}-68,019,840 r^{5}-21,915,328 r^{6}\right) / 33$ \\
& $h_{2}=\left(5,467,200 r^{2}+32,499,936 r^{3}+68,439,424 r^{4}+63,161,280 r^{5}+21,792,896 r^{6}\right) / 33$ \\
& $h_{3}=\left(-262,4256 r^{2}-16,392,096 r^{3}-38,323,552 r^{4}-38,868,480 r^{5}-14,446,976 r^{6}\right) / 33$ \\
& $h_{4}=\left(70,752 r^{2}+450,072 r^{3}+1,104,880 r^{4}+1,214,640 r^{5}+489,728 r^{6}\right) / 3$ \\
& $h_{5}=\left(-102,912 r^{2}-663,264 r^{3}-1,673,104 r^{4}-1,943,424 r^{5}-857,024\right) / 33$ \\
\hline \hline
\end{tabular}

where $z_{1}=\omega h_{1} / M_{1}$ and $z_{2}=\omega h_{2} / M_{2}, A(z), B(z), C(z)$, and $D(z)$ are as already defined, and

$$
\begin{aligned}
& G(z)=\sum_{d}^{N_{g}} g_{k}(i z)^{k}+\sum_{d}^{N_{h}} \frac{h_{k}}{E^{+}(z)^{k}} \\
& H(z)=\sum_{k}^{N_{g}} g_{k}(i z)^{k}+\sum_{d}^{N_{h}} \frac{h_{k}}{E-(z)^{k}}
\end{aligned}
$$

and note that $G\left(z_{1}\right)=G\left(r z_{2}\right)$ and $H\left(z_{1}\right)=H\left(r z_{2}\right)$, where $r$ is the interface ratio,

$$
r=h_{1} M_{2} / h_{2} M_{1}
$$

An exact interface condition, therefore, would require that $D\left(z_{2}\right)=0$, and

$$
\left[G\left(r z_{2}\right)+\frac{A\left(r z_{2}\right) H\left(r z_{2}\right)}{B\left(r z_{2}\right)}\right]=C\left(z_{2}\right)
$$

Thus, we pick the coefficients $c_{k}$ and $d_{k}$ as before to force to zero coefficients in the Taylor series of $D(z)$ about $z=0$. The coefficients $a_{k}$ and $b_{k}$ needed to find $A(z)$ and $B(z)$ are determined once a downwind boundary condition for $u_{N_{1}}$ is chosen. Thus, for a particular scheme, these coefficients are all identical to those already given in Table 1 . The coefficients $g_{k}$ and $h_{k}$ are then chosen to zero coefficients in the Taylor series of

$$
\left[G\left(r z_{2}\right)+\frac{A\left(r z_{2}\right) H\left(r z_{2}\right)}{B\left(r z_{2}\right)}\right]-C\left(z_{2}\right)
$$

about $z_{2}=0$. Note that it is not necessary to match the order of accuracy of the downwind boundary conditionfor $u_{N_{1}}$ and the upwind interface condition for $v_{0}$, though $D\left(z_{2}\right)$ and

$$
\left[G\left(r z_{2}\right)+\frac{A\left(r z_{2}\right) H\left(r z_{2}\right)}{B\left(r z_{2}\right)}\right]-C\left(z_{2}\right)
$$

should be zero to the same order of accuracy.

Table 3 gives the coefficients $g_{k}$ and $h_{k}$, which correspond to several of the upwind conditions given there. These can be used with any of the downwind conditions for $u_{N_{1}}$ so long as the order of accuracy of the downwind condition is equal to or greater than the order of accuracy of the upwind interface condition.

Figure 7 shows the results of two numerical experiments with the interface conditions 4.1. In each case, the results obtained using the interface conditions discussed are compared with a scheme where the interface is handled in a conventional way, by using a finite difference approximation across the interface: the corresponding Pade derivative with unequal grid spacing on a three-point stencil ( 1 point at the interface and 1 point on each side) is formally thirdorder accurate. For both approaches, upwind scheme 1.1 is used for point $u_{0}$ and downwind scheme 1.1 is used for point $v_{N_{2}}$.

In the first test, the advection speed is $M_{1}=2$ on the left-hand side of the interface and $M_{1}=1$ on the right-hand side, simulating the passage of the wave through a shock. The wave is very well resolved with $N_{1}=N_{2}=200$ [about 50 points across the pulse,
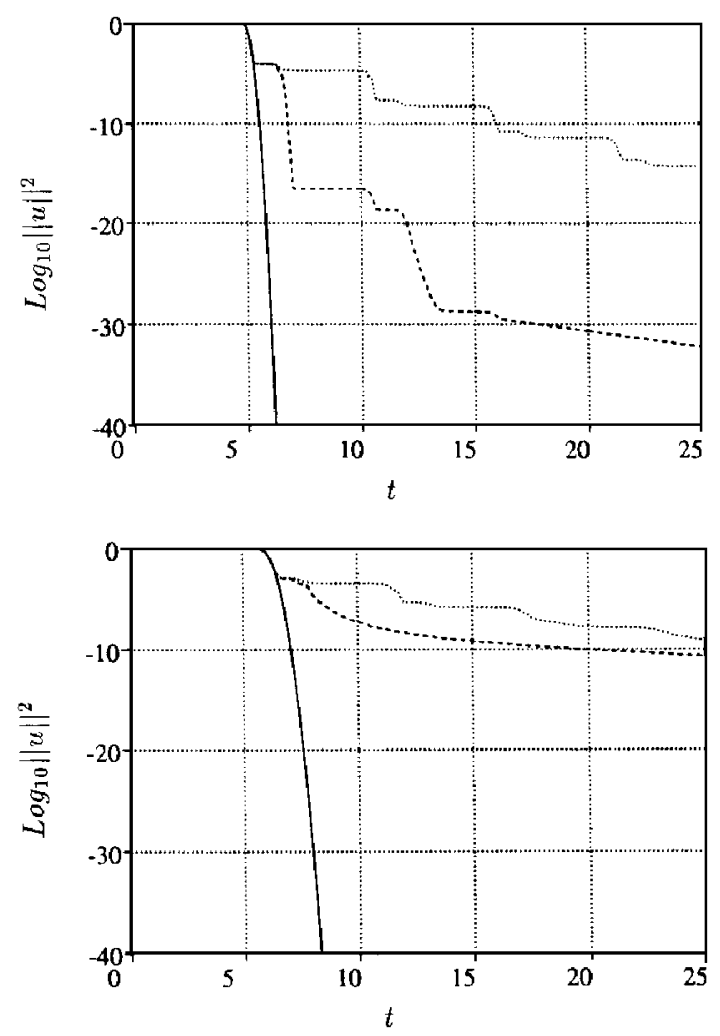

Fig. 7 Energy left in the computational domain as a function of time; comparison of present interface technique and conventional finite difference treatment: - $\longrightarrow$, exact solution; - - - present approach; and .... conventional approach. See text for description of the tests.

which is initially centered on the right-hand side and given by $u=$ exp[-8(x-2) $\left.)^{2}\right], C=1$, and $L_{1}=L_{2}=4$. In Fig. 7, the sum of the $L 2$ norm of $u$, defined here as

$$
\|u\|^{2}=\frac{1}{M_{1}} \sum_{d}^{N_{1}} u_{j}^{2}+\frac{1}{M_{2}} \sum_{j}^{N_{2}} v_{j}^{2}
$$

for both domains, is plotted as a function of time. The conventional interface treatment leads to much larger reflections of the sawtooth waves from the interface. In the second test, the grid spacing is doubled across the interface. In this case, $M_{1}=M_{2}=1$ and $L_{1}=L_{2}=4$, and $N_{1}=100$ points are used on the left ( 25 points across the pulse) and $N_{2}=50$ points are used on the right (12.5 points across the pulse). Note that the time step is the same for both domains; therefore, the CFL number on the left is 1 and on the right is 0.5 . Thus, the highest frequencies (which are not removed by the boundary conditions or interface) are damped only very slowly (the RK4 scheme is less dissipative for the high frequenciesat low CFL number). In both tests, the new interface condition is better able to remove spurious energy from the domain for long times. 


\section{Implementation for the Inviscid Flow Equations}

The boundary conditions for the one-dimensionalmodel equation can be applied to the linearized equations of gasdynamics. They can further be applied to nonlinear computations, provided that the equations are linearized near the boundary when this is appropriate to the particular problem being solved. ${ }^{3}$ In the one-dimensional case, the linearized equations can be reduced to a system of three independent (characteristic) equations of the form of Eq. (1). The three equations represent two acoustic waves and an entropy wave with advection speeds $s$ equal to $M+1, M_{-} 1$, and $M$, respectively, where $M$ is the Mach number of the (uniform) freestream to which the disturbances are added. In a subsonic flow, therefore, waves can propagate to both the left and the right. These boundary conditions presented assume that the wave travels to the right and need to be slightly altered as follows. For the right-hand side ( $x=1$ here), the boundary condition is

$$
\sum_{N_{a}}^{N_{a}} q_{k}\left(\frac{h}{s}\right)^{k} \frac{\mathrm{d}^{k} u_{N}}{\mathrm{~d} t^{k}}+\beta * \frac{h}{s} \frac{\mathrm{d} u_{N \_1}}{\mathrm{~d} t}=\sum_{d}^{N_{b}} r_{k} u_{N \_k}
$$

where if $s>0$,

$$
q_{k}=a_{k}, \quad r_{k}=b_{k}, \quad \beta *=\beta
$$

and if $s<0$,

$$
q_{k}=\left(-^{1}\right)^{k} c_{k}, \quad r_{k}=d_{k}, \quad \quad \beta *=-\beta
$$

whereas for the left-hand side ( $x=0$ here),

$$
\sum_{-}^{N_{a}} q_{k}\left(\frac{h}{s}\right)^{k} \frac{\mathrm{d}^{k} u_{0}}{\mathrm{~d} t^{k}}+\beta * \frac{h}{s} \frac{\mathrm{d} u_{1}}{\mathrm{~d} t}=\sum_{d}^{N_{b}} r_{k} u_{k}
$$

where if $s>0$

$$
q_{k}=c_{k}, \quad r_{k}=d_{k}, \quad \quad \beta *=\beta
$$

and if $s<0$

$$
q_{k}=(-1)^{k} a_{k}, \quad r_{k}=b_{k}, \quad \beta *=\_\beta
$$

The constants $a_{k}, b_{k}, c_{k}$, and $d_{k}$ are as given in Tables 1 and 2. If $s=0$, the correct boundary condition is simply $\partial u_{N} / \partial t=0$.

Finally, the analysis presented here can be effectively extended to tackle the multidimensional problem directly. These results will be presented in future publications.

\section{Acknowledgments}

The author wishes to thank Clarence Rowley for his assistance in generating the data presented in Sec. IV and Jon Freund for his helpful remarks on this work.

\section{References}

${ }^{1}$ Enquist, B., and Majda, A., "Absorbing Boundary Conditions for the Numerical Simulation of Waves," Mathematics of Computation, Vol. 31, No. 139, 1977, pp. 629-651.

${ }^{2}$ Giles, M. B.,"Non-Reflecting Boundary Conditions for Euler Equation Computations," AIAA Journal, Vol. 28, No. 12, 1990, pp. 2050-2058.

${ }^{3}$ Colonius, T., Lele, S. K., and Moin, P., "Boundary Conditions for Direct Computation of Aerodynamic Sound," AIAA Journal, Vol. 31, No. 9, 1993 , pp. 1574-1582.

${ }^{4}$ Bayliss, A., and Turkel, E., "Outflow Boundary Conditions for Fluid Dynamics," SIAM Journal on Scientific and Statistical Computing, Vol. 3, No. 2, 1982, pp. 250-259.

${ }^{5}$ Trefethen, L. N., "Group Velocity in Finite Difference Schemes," SIAM Review, Vol. 24, No. 2, 1982, pp. 113-136.
${ }^{6}$ Vichnevetsky, R., "Wave Propagation Analysis of Difference Schemes for Hyperbolic Equations: A Review," International Journal for Numerical Methods in Fluids, Vol. 7, No. 5, 1987, pp. 409-452.

${ }^{7}$ Tam, C. K. W., and Webb, J. C., "Radiation Boundary Condition and Anisotropy Correction for Finite-Difference Solutions of the Helmholtz Equation," Journal of Computational Physics, Vol. 113, No. 1, 1994, pp. $122-133$.

${ }^{8}$ Hall, K. C., Lorence, C. B., and Clark, W. S., "Nonreflecting Boundary Conditions for Linearized Unsteady Aerodynamic Calculations," AIAA Paper 93-0882, Jan. 1993.

${ }^{9}$ Huh, K. S., Agarwal, R. K., and Widnall, S. E., "Numerical Simulation of Acoustic Diffraction of Two-Dimensional Rigid Bodies in Arbitrary Flows," AIAA Paper 90-3920, Oct. 1990.

${ }^{10} \mathrm{Tam}$, C. K. W., and Webb, J. C., "Dispersion-Relation-Preserving Finite Difference Schemes for Computational Acoustics," Journal of Computational Physics, Vol. 107, No. 2, 1993, pp. 262-281.

${ }^{11}$ Colonius, T., Lele, S. K., and Moin, P., "The Scattering of Sound Waves by a Vortex: Numerical Simulations and Analytical Solutions," Journal of Fluid Mechanics, Vol. 260, Feb. 1994, pp. 271-298.

${ }^{12}$ Mitchell, B. E., Lele, S. K., and Moin, P., "Direct Computation of the Sound Generated by Vortex Pairing in an Axisymmetric Jet," AIAA Paper 95-0504, Jan. 1995.

${ }^{13}$ Mitchell, B. E., Lele, S. K., and Moin, P., "Direct Computation of the Sound from a Compressible Co-Rotating Vortex Pair," Journal of Fluid Mechanics, Vol. 285, Feb. 1995, pp. 181-202.

${ }^{14}$ Agarwal, R. K., and Huh, K. S., "A Dispersion-Relation Preserving Fourth-Order Compact Time-Domain/Frequency-Domain Finite-Volume Method for Computational Acoustics," AIAA Paper 96-0277, Jan. 1996.

${ }^{15}$ Colonius, T., Lele, S. K., and Moin, P., "Sound Generation in a Mixing Layer,” Journal of Fluid Mechanics, Vol. 330, Jan. 1997, pp. 375-409.

${ }^{16}$ Freund, J. B., Lele, S. K., and Moin, P., "Direct Simulation of a Supersonic Round Turbulent Shear Layer," AIAA Paper 97-0760, Jan. 1997.

${ }^{17}$ Lele, S. K., "Computational Aeroacoustics: A Review," AIAA Paper 97-0018, Jan. 1997.

${ }^{18}$ Vichnevetsky, R., "High Order Numerical Sommerfeld Boundary Conditions: Theory and Experiments," Computers and Mathematics with Applications, Vol. 11, No. 1-3, 1985, pp. 67-78.

${ }^{19}$ Lele, S. K., "Compact Finite Difference Schemes with Spectral-Like Resolution," Journal of Computational Physics, Vol. 103, No. 1, 1992, pp. $16-42$

${ }^{20} \mathrm{Kim}$, J. W., and Lee, D. J., "Optimized Compact Finite Difference Schemes with Maximal Resolution," AIAA Journal, Vol. 34, No. 5, 1996, pp. 887-893.

${ }^{21} \mathrm{Hu}$, F. Q., Hussaini, M. Y., and Manthey, J., "Application of Low Dissipation and Dispersion Runge-Kutta Schemes to Benchmark Problems in Computational Aeroacoustics," NASA TR CP-3300, May 1995.

${ }^{22}$ Carpenter, M. H., Gottlieb, D., and Ararbanel, S., "The Stability of Numerical Boundary Treatments for Compact High-Order Finite-Difference Schemes," Journal of Computational Physics, Vol. 108, No. 2, 1993, pp $272-295$.

${ }^{23}$ Vichnevetsky, R., and Parsier, E. C., "Nonreflecting Upwind Boundaries for Hyperbolic Equations," Numerical Methods for Partial Differential Equations, Vol. 2, No. 1, 1986, pp. 1-12.

${ }^{24}$ Trefethen, L. N., "Group Velocity Interpretation of the Stability Theory of Gustafsson, Kreiss, and Sundström," Journal of Computational Physics, Vol. 49, No. 2, 1983, pp. 199-217.

${ }^{25}$ Gustafsson, B., Kreiss, H.-O., and Sundstrom, A., "Stability Theory of Difference Approximations for Mixed Initial Boundary Value Problems. II," Mathematics of Computation, Vol. 26, No. 119, 1972, pp. 649-686.

${ }^{26}$ Wolfram, S., Mathematica, 2.2 ed., Wolfram Research, Inc., Champaigne, IL, 1994.

${ }^{27}$ Warming, R. F., and Beam, R. M., "Stability of Semidiscrete Approximations for Hyperbolic Initial-Boundary-Value Problems: An Eigenvalue Analysis," Lecture Notes in Physics, Vol. 264, Springer-Verlag, New York, 1986, pp. 647-653.

${ }^{28}$ Cain, A. B., and Bush, R. H., "Numerical Wave Propagation Analysis for Stretched Grids,” AIAA Paper 94-0172, Jan. 1994. 